\title{
Coupling of the accretion disk and corona around black holes
}

\author{
B. F. Liu \\ National Astronomical Observatories, Chinese Academy of Sciences, \\ 20A Datun Road, Chaoyang District, Beijing 100012, China \\ email: bfliu@nao.cas.cn
}

\begin{abstract}
The interaction between a hot corona and a cold thin disk can drive cold gas evaporating into the corona or hot gas condensing into the thin disk. The evaporation is caused by heat conduction downwards to the disk; while condensation is caused by overcooling of corona gas through inverse Compton scattering. Evaporation occurs at low accretion rates when the corona cannot efficiently radiate the viscous heat, thereby part of which is conducted down and heats up gas. Condensation occurs at high accretion rates when the Compton cooling of disk photons is strong enough to efficiently cool the corona gas. An important consequence of the evaporation is complete removal of the disk at a certain distances. In contrast, condensation can lead to a weak corona or complete collapse of the corona. This causes the observed transitions between various spectral states, at which the accretion flows are dominated respectively by ADAF, disk+ corona, and thin disk from low to high accretion rates, providing a natural explanation to the low, intermediate and high spectral states in BHXRBs, as well as their transitions. The same process can also be applied to accretion around supermassive black holes.
\end{abstract}

Keywords. Accretion, accretion disks — black hole physics - X-rays: binaries - X-rays:galaxiesgalaxies: active

\section{Introduction}

The spectral behavior of black hole X-ray binaries (BHXRBs) exhibits differing states and transitions. It is commonly believed that the accretion is dominantly via an ADAF at low/hard states and a thin disk at high/soft states. The transition can be triggered by heat conduction, thermal instability or "strong ADAF principle". Among these models the conduction-caused evaporation is the only one which can naturally interpret most of the phenomena relevant to the spectral variation and quantitatively fits observational spectra. The model can be applied to supermassive black holes (SMBHs) and interprets some of the observations such as spectra in low-luminosity active galactic nuclei (AGNs) and existence of true Seyfert 2 galaxies. In this talk, I introduce the model and show the predictions and comparison with observations.

\section{The disk corona model}

In our disk corona model, it is assumed that an optically thick and geometrically thin accretion disk is embedded in an optically thin and geometrically thick accretion corona around a black hole (see Figure 1). Viscous heat produced in the corona is partially conducted down due to a large temperature between the disk and corona. Photons emitted by the thin disk going through the corona can be Compton scattered in the corona. The dynamical and energy interaction in the coupled disk and corona results in mass exchange, either the disk gas evaporating into the corona, or the corona gas condensing to the disk, as illustrated in Figure 1. The accretion is thus via both a thin disk and a 


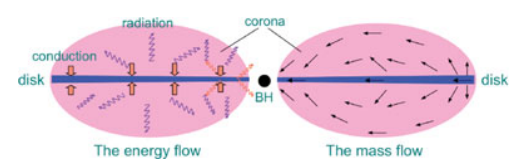

Figure 1. Schematic description of the interaction of the accretion disk and the corona. The left part shows how the energy exchanges between the disk and corona; The right part shows how the mass flows to the black hole.

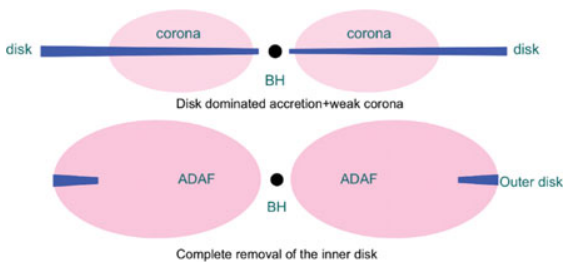

Figure 2. Consequences of disk corona interaction. At low accretion rates, the disk is truncated by the evaporation, leading to an ADAF dominated, hard state spectrum; while at high accretion rates, a full thin disk coexists with a weak corona.

corona, with mass continuously evaporating or condensing between the disk and corona. The relative strength of the disk and corona depends on the Eddington-scaled accretion rate. The extreme case is complete evaporation of the thin disk when the accretion rate is very low, or complete condensation of corona due to strong Compton cooling at very high accretion rates, as shown in Figure 2.

Detailed investigations of the interaction between the disk and the corona reveal that the accretion flow continuously changes its form with the value of mass supplying rates to the disk. As described in Figure 3, the accretion at low mass accretion rates is via an ADAF connecting to an outer disk (for details see Meyer et al. 2000a; Liu et al. 2002a). Therefore, the emission is dominated by the ADAF, and a quiescent or hard state spectrum forms (Meyer at al. 2000b). At an accretion rate around 1\% Eddington value, an inner accretion disk can be present, feeding by the condensation of corona gas (Liu et al. 2006; Meyer et al. 2007; Liu et al. 2007; 2011) The disk contribution increases with increase of accretion rate, and the spectrum changes from hard to intermediate state (Taam et al. 2008; Qiao \& Liu 2010). At high accretion rates $(\dot{m}>0.03)$, the evaporation is no longer strong enough to completely remove the disk. Instead, large disk radiations can cool the corona through inverse Compton scattering, leading to efficient condensation of corona gas ( Liu et al. 2007; Meyer-Hofmeister et al. 2012). This weakens the corona and the radiation is dominated by the disk, forming the soft state. At even high accretion rates, corona can not survive any more as a consequence of Compton cooling. Nevertheless, additional heating to the corona, such as magnetic reconnection (Liu et al. 2002b;2003; Cao 2009), could support the corona, forming a hard X-ray tail or so-called very high state (Liu et al. 2012).

\section{Comparison with observations}

The prediction of accretion flow varying with the mass accretion rates, as a consequence of disk corona interaction, has naturally interpreted many complex observational phenomena in both BHXRBs and AGNs as follows.

- The low/hard, intermediate, and high/soft spectral states in BHXRBs and types of AGNs shown in Figure 3 are caused by evaporation/condensation.

- Hystereses between the hard-to-soft transition and soft-to-hard transition (Liu et al. 2005; Meyer-Hofmeister et al. 2005) and "q-curve" (e.g.Dunn et al. 2008) in the hardnessintensity diagram (Meyer et al. 2009) are interpreted as different strength of Compton cooling at different evolutionary history, see Figure 4. 


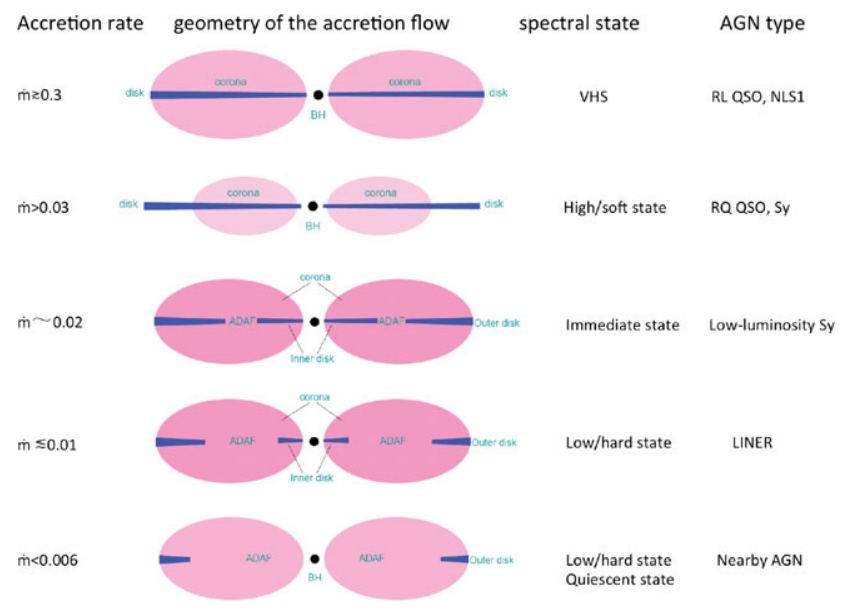

Figure 3. The variation of the accretion flow with the accretion rate predicted by the disk corona interaction model. Corresponding spectral states of black hole X-ray binaries and AGN types are also shown.
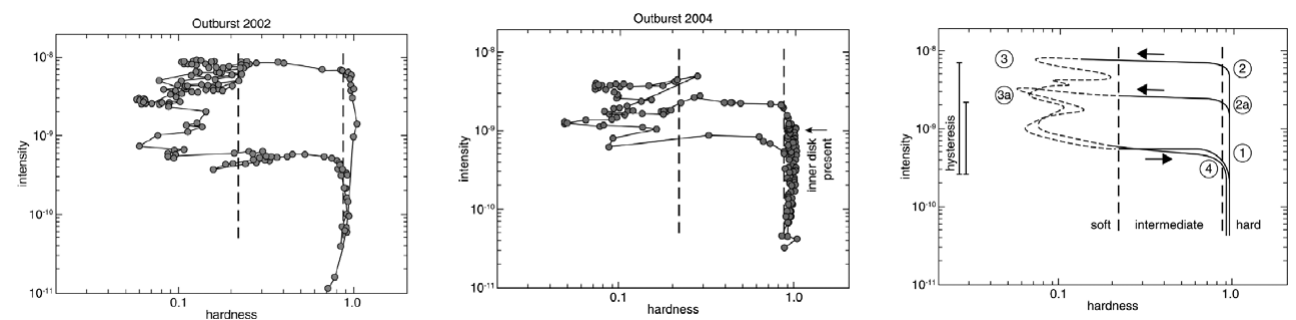

Figure 4. Observed outbursts of GX339-4 (left and middle panels) and interpretation (right panel) by the disk corona model (Meyer-Hofmeister et al. 2009).

- The weak disk component in addition to the ADAF spectrum at low/intermediate state of BHXRBs is from a weak inner disk feeding by the condensation of corona gas (Liu et al. 2006; 2007; Meyer et al. 2007).

- The disk truncation as a function of Eddington ratio in the low/hard state in AGNs is self-consistently determined by evaporation (Liu et al. 1999; Taam et al. 2012), as shown in Figure 5.

- Negative correlation between Eddington ratio and hard X-ray photon index for BHXRBs at low/hard state (e.g. XTE J1180+480, Qiao \& Liu 2010) and positive correlation at intermediate/high state (Qiao \& Liu 2012) are caused by the variation of Compton cooling.

- Disappearance of broad line region at low luminosity AGNs and existence of true Seyfert 2 galaxies are a consequence of removal of the thin disk and hence the broad line region (Liu \& Taam 2009).

Further investigations of the coupling between the disk and the corona reveal that the critical accretion rate for a change of accretion modes depends on the viscosity (Qiao \& Liu 2009), and the truncation radius depends also on the magnetic field (Qian et al. 2007). Comparisons with observations give constraints to the value of viscosity parameter and the strength of magnetic field ( Liu et al. 2012).

In the case of luminous AGN, the corona is very weak as a result of efficient Compton cooling (Liu et al. 2012; Meyer-Hofmeister et al. 2012). We find that magnetic field should 


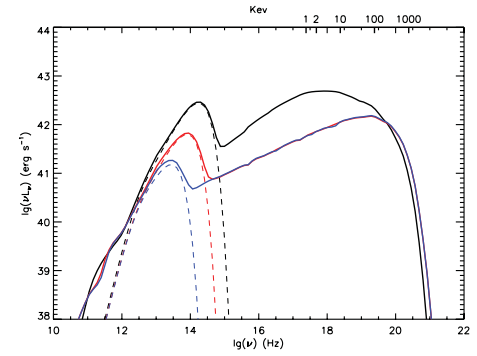

Figure 5. The spectral energy distribution from an inner ADAF and truncated disk around a SMBH of $M=10^{8} M_{\odot}$ at low accretion rates with truncation radius determined by the evaporation (Taam et al. 2012).

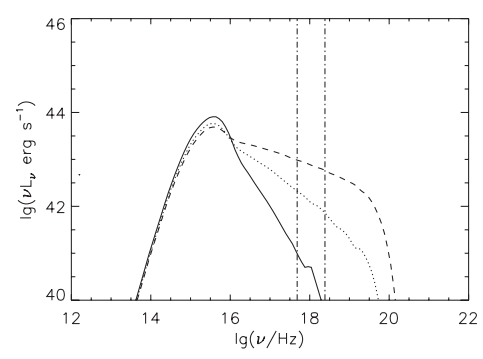

Figure 6. The spectral energy distribution from a full thin disk and corona around a SMBH of $M=10^{8} M_{\odot}$ at high accretion rates, with a fraction $(f)$ of accretion energy released in the corona, $f=0.3,0.2,0.1$ from upper to lower curves (Liu et al. 2012).

play an important role in transferring accretion energy to the corona, leading to strong X-ray radiations (Liu et al. 2012), as shown in Figure 6.

\section{Conclusion}

I present a coupled disk corona model which has been studied for more than 10 years, and show that it can interpret most of the observational features in BHXRBs and AGNs.

\section{References}

Cao, X. 2009, MNRAS, 394, 207

Dunn, R. J. H., Fender, R. P., Körding, E. G., Cabanac, C., \& Belloni, T.: 2008, MNRAS, 387, 545

Liu, J. Y., Liu, B. F., Qiao, E. L., \& Mineshige, S., 2012, ApJ, 754:81

Liu, B. F., Yuan, W., Meyer, F., Meyer-Hofmeister, E., \& Xie, G. Z. 1999, ApJ, 527, L17

Liu, B. F., Mineshige, S., Meyer, F., Meyer-Hofmeister, E., \& Kawaguchi, T. 2002a, ApJ, 575, 117

Liu, B. F., Mineshige, S., \& Shibata, K. 2002b, ApJ, 572, L173

Liu, B. F., Meyer, F., \& Meyer-Hofmeister, E. 2005, A\&A, 442, 555

Liu, B. F., Meyer, F., \& Meyer-Hofmeister, E. 2006, A\&SA, 454, L9

Liu, B. F., Taam, R. E., Meyer-Hofmeister, E., \& Meyer, F. 2007, ApJ, 671, 695

Liu, B. F. \& Taam, R. E., 2009, ApJ, 707, 233

Liu, B. F., Done, C., \& Taam, 2011, ApJ, 726:10

Liu, B. F. \& Taam, R. E., 2012, in preparation.

Meyer, F., Liu, B. F., \& Meyer-Hofmeister, E. 2000a, A\&A, 361, 175

Meyer, F., Liu, B. F., \& Meyer-Hofmeister, E. 2000b, $A \mathscr{E} A$,354, L67

Meyer, F., Liu, B. F., \& Meyer-Hofmeister, E. 2007, A\&A, 463, 1

Meyer-Hofmeister, E., Liu, B. F., \& Meyer, F. 2005, A\&AA, 432, 181

Meyer-Hofmeister, E., Liu, B. F., \& Meyer, F. 2009, A\&A A, 508, 329

Meyer-Hofmeister, E., Liu, B. F., \& Meyer, F. 2012, A\& A, 544, A87

Qian, L., Liu, B. F., \& Wu, X. B., 2007, ApJ, 668, 1145

Qiao, E. \& Liu, B. 2009, PASJ, 61, 403

Qiao, E. \& Liu, B. 2010, PASJ, 62, 661

Qiao, E. \& Liu, B. F. 2012, ApJ 744:145

Taam, R. E., Liu, B. F., Meyer, F., \& Meyer-Hofmeister, 2008, ApJ, 688, 527

Taam, R. E., Liu, B. F., Yuan, W., \& Qiao, E. 2012, ApJ, in press 